\title{
Estado nutricional de hierro, cobre y zinc en relación con el uso de método anticonceptivo hormonal
}

\author{
Iron, copper and zinc status in \\ relation with the hormonal \\ contraceptive method
}

\begin{abstract}
Childbearing age women are part of a vulnerable group to present micronutrient deficiencies. It has been associated the contraceptive method (CM) with iron (Fe) status, and the hormonal CM (HCM) with the alteration of copper (Cu) and zinc $(\mathrm{Zn})$ biomarkers. The aim of this study was to assess the effect of $C M$ over the biomarkers of $\mathrm{Fe}, \mathrm{Cu}$ and $\mathrm{Zn}$ status, and the prevalence of micronutrient deficiency on 81 Chilean childbearing age women. We evaluated mean corpuscular volume (MCV), hemoglobin (Hb), zinc protoporphyrin (Zpp), serum $\mathrm{Fe}(\mathrm{FeS})$, transferrin saturation (TS), total Fe binding capacity (TIBC), transferrin receptor (Tfr), serum ferritin (SF), serum copper (CuS), ceruloplasmin (Cp) mass, activity and specific activity and serum zinc ( $\mathrm{ZnS}$ ). From the total women, $40 \%$ used HCM and $49 \%$ did not use HCM. Women using HCM showed higher values of FeS, ST, CuS, Cp mass and activity $(p<0.05)$ and lower Zpp $(p<0.05)$ compared with those that did not use HCM. Not statistical differences were found on MCV, Hb, TIBC, Tfr, SF, ZnS and Cp specific activity values between groups. HCM utilization did not affect $\mathrm{Fe}, \mathrm{Cu}$ and $\mathrm{Zn}$ status in the studied population.
\end{abstract}

Key words: Iron, copper, zinc, contraceptive method, childbearing age women.

\section{INTRODUCCIÓN}

El hierro (Fe), cobre (Cu) y zinc ( $\mathrm{Zn}$ ) son minerales esenciales para el ser humano, están involucrados en varios procesos metabólicos. El Fe se encarga del transporte y almacenaje de oxígeno, fosforilación oxidativa, metabolismo de neurotransmisores y síntesis de ADN; el $\mathrm{Cu}$ es componente de varias enzimas, está involucrado en el transporte de electrones, dismutación de superóxido, síntesis de melanina, entrecruzamiento de colágeno y elastina, síntesis de neurotransmisores, oxidación y homeostasis de Fe, mientras que el $\mathrm{Zn}$ participa como cofactor de diversas enzimas, estructura de las proteínas y en la regulación de la expresión de genes $(1,2)$.

Las mujeres en edad reproductiva conforman un grupo vulnerable de presentar deficiencia de micronutrientes, principalmente en países en vía de desarrollo. En éste grupo poblacional, los requerimientos de Fe se encuentran aumentados por las pérdidas de éste mineral durante la menstruación (2). La anemia es el principal indicador de deficiencia de Fe, según la encuesta de salud realizada en Chile el año 2003,
Angélica Borja-Magno María Fernanda Mujica-Coopman Fernando Pizarro A. Manuel Olivares G.

Laboratorio de Micronutrientes, Instituto de Nutrición y Tecnología de los Alimentos (INTA), Universidad de Chile. Santiago, Chile.

Dirigir la correspondencia a: Angélica Irais Borja Magno Eduardo Farfán No.788 Colonia: Los Eucaliptos Irapuato, Guanajuato, México. E-mail: angelica_borja@hotmal.com

Este trabajo fue recibido el 6 de Mayo de 2014 y aceptado para ser publicado el 24 de Junio de 2014.
5,1\% de las mujeres en edad reproductiva la presentaban (3). A su vez, Cediel y cols., mostraron en mujeres chilenas entre 20 y 55 años, que 5,9 y 25\% presentaron deficiencia Cu y Zn, respectivamente (4).

El método anticonceptivo $(M A)$ se ha relacionado con el estado nutricional de Fe, debido a su influencia sobre las pérdidas menstruales. El uso de MA de tipo hormonal (MAH) disminuye las pérdidas menstruales hasta 50\%, mientras que se ha sugerido que el uso del dispositivo intrauterino (DIU) las incrementa al doble (5). Las pérdidas menstruales son la principal fuente de variación de los requerimientos de Fe en este grupo de la población (6). Por lo tanto, se ha sugerido que las mujeres que utilizan $\mathrm{MAH}$ presentan mejor estado nutricional de Fe que aquellas que utilizan DIU (7) o que aquellas que no utilizan ningún MA (8).

Por otra parte, estudios en mujeres que utilizan $\mathrm{MAH}$ han mostrado un incremento en la concentración de cobre sérico (CuS), ceruloplasmina masa (Cp masa) y actividad (Cp actividad) $(9,10)$, el mecanismo que se ha propuesto indica 
que la administración de estrógenos influye en la producción y liberación de ceruloplasmina a nivel hepático (10). A su vez, se ha descrito que la actividad específica (Cp actividad/ Cp masa) no se alteraría por terapia hormonal $(11,12)$. Respecto al Zn, existe controversia del efecto del MAH. Algunos estudios muestran una disminución en la concentración de ZnS en mujeres que utilizan MAH (13), esto se ha atribuido a que la administración de $\mathrm{MAH}$ puede reducir la concentración de albúmina, proteína a la cual se une $75 \%$ del Zn plasmático (14). Mientras que otros no observaron diferencias al comparar con mujeres que no los utilizan (10)

Actualmente en Chile, según la Encuesta de Calidad de vida y Salud del 2006, 13,6\% de las mujeres indicó usar el dispositivo intrauterino (DIU), 18\% utilizó MAH de tipo oral e inyectable y $65,4 \%$ utilizaba preservativos, estaba esterilizada o no utiliza ningún método (15).

El objetivo del estudio fue evaluar el efecto del MA sobre los biomarcadores del estatus de Fe, Cu y Zn y la prevalencia de la deficiencia de estos micronutrientes en mujeres chilenas en edad reproductiva.

\section{SUJETOS Y MÉTODOS}

Sujetos. Se conformó una muestra por conveniencia de 81 mujeres en edad reproductiva (18 a 45 años), procedentes de la comuna de Macul, Santiago, Chile, quienes fueron evaluadas entre julio y agosto del año 2013. Las participantes no presentaban antecedentes de consumo de suplementos de vitaminas o minerales 6 meses previos al estudio. Además no presentaban diagnóstico de embarazo ni se encontraban amamantando al momento del estudio. Los grupos fueron conformados en base al método anticonceptivo empleado.

Mediciones antropométricas. El peso y talla se midieron por técnicas descritas, 16 utilizando una balanza $(0,1 \mathrm{~kg}$ de precisión) y tallímetro (1 $\mathrm{cm}$ de precisión) marca SECA. El estado nutricional se determinó mediante el cálculo del índice de masa corporal (IMC) y según el criterio de la Organización Mundial de la Salud (OMS) las participantes se clasificaron con bajo peso (IMC $\leq 18,5)$, estado nutricional normal (IMC $18,5-24,9 \mathrm{~kg} / \mathrm{m}^{2}$ ), sobrepeso (IMC $\left.25,0-29,9 \mathrm{~kg} / \mathrm{m}^{2}\right) \mathrm{u}$ obesidad $\left(\geq 30 \mathrm{~kg} / \mathrm{m}^{2}\right)$.

Parámetros bioquímicos. A cada participante se le extrajo $15 \mathrm{ml}$ de sangre venosa posterior a un ayuno nocturno, entre las 8 - 9 AM. Se determinó hemoglobina $(\mathrm{Hb})$, volumen corpuscular medio (VCM) (CELL-DYN 3200, ABBOTT Diagnostics, Abbott Park, IL), hierro sérico (FeS), capacidad total de unión de hierro (TIBC) y saturación de transferrina (ST) (17), Zinc protoporfirina (Zpp) (Hematofluorómetro modelo 206D, AVIV Biomedical Inc., Lakewood, NJ), ferritina sérica (FS) (18), receptor de transferrina (rTf) (Enzyme-linked immunosorbent assay, Ramco Laboratories Inc.,Houston, TX, USA). Zinc (ZnS) y cobre (CuS) sérico (Espectrómetro de absorción atómica, modelo 2280, The Perkin-Elmer Corporation, Norwalk, CT, USA), concentración de Ceruloplasmina inmunoreactiva (Cp masa) (Array Protein System, Beckman Instruments Inc., Brea, CA, USA). Y la actividad de la Ceruloplasmina ( $\mathrm{Cp}$ actividad) (Método $\mathrm{p}$-phenylenediamine oxidasa) (19). Se calculó la Actividad específica de Cp (Cp actividad/Cp masa).

La anemia se definió como $\mathrm{Hb}<12 \mathrm{~g} / \mathrm{dL}$ (20), Anemia por deficiencia de hierro (ADF) como anemia y 2 o más indicadores alterados; deficiencia de hierro sin anemia (DFSA) como $\mathrm{Hb}$ normal y dos o más indicadores hematológicos alterados; depósitos depletados de Fe (DDF) como FS bajo lo normal. Los puntos de corte utilizados fueron VCM<80fL, Zpp $>70 \mu \mathrm{g}$ /
dL GR, ST <15\%, rTf >8,6 y FS<15 $\mu$ g/L (20-22).

La deficiencia de Zn se definió como ZnS por debajo de $70 \mu g / d L$ y deficiencia de Cu, como de CuS por debajo de 80 $\mu g / d L(23)$.

Ingesta dietética. La ingesta dietética se evaluó utilizando una encuesta de frecuencia de consumo (EFC), se emplearon utensilios comunes de cocina para estimar las porciones. La ingesta dietética fue convertida a datos de ingesta de micro y macronutrientes, utilizando la Tabla de Composición de Alimentos de Chile (24), y se complementó con datos de los alimentos comúnmente consumidos en Chile (25), y con la base de datos nacionales de nutrientes estándar de referencia del Departamento de Agricultura de Estados Unidos (USDA) (26).

Análisis estadístico. Los datos que presentaron distribución normal se expresaron en medias y desviación estándar (DE), mientras que aquellos que no presentaban distribución simétrica, se transformaron a sus logaritmos naturales, posteriormente se calculó el anti-logaritmo y se expresaron en promedio geométrico y rango de $\pm 1 \mathrm{DE}$. Se empleó el test estadístico $\mathrm{T}$ de Student de dos colas no pareado para comparar las características generales de los sujetos y T de Student de una cola no pareado para comparar los biomarcadores de Fe, Cu y Zn. Para comparar prevalencias se utilizó el test estadístico Chi cuadrado. Se utilizó el programa STATA 12,0 (StataCorp, College Station, TX, USA) para el análisis de los datos. Todas las comparaciones tuvieron un nivel de significación de 0,05.

Consideraciones éticas. Este estudio fue aprobado por el Comité de Ética del Instituto de Nutrición y Tecnología de los Alimentos (INTA) de la Universidad de Chile de acuerdo a la declaración de Helsinki y el código de Nuremberg. Todas las mujeres participaron voluntariamente después de leer y firmar el consentimiento informado.

\section{RESULTADOS}

Se evaluaron 81 mujeres en edad reproductiva, 40\% $(n=32)$ utilizaba MAH (grupo MAH+), 49\% $(n=40)$ utilizaban preservativo o se encontraban esterilizadas o no empleaban ningún MA (grupo MAH-), 10\% ( $n=8)$ usaba DIU y $1 \%(n=1)$ anillo vaginal, debido al reducido número de participantes que conformaban el grupo DIU y anillo vaginal, no se incluyeron en el análisis. Por lo que sólo se realizó la comparación entre los grupos $\mathrm{MAH}+$ y $\mathrm{MAH}-$. De las mujeres que conformaron el grupo $\mathrm{MAH}+, 72 \%$ empleaba $\mathrm{MAH}$ oral, $19 \%$ inyectable y 9\% implante hormonal.

Las características generales de las participantes se muestran en la tabla 1. Del total de las participantes, la edad promedio fue de 33,4 \pm 9,0 años. Se observó que en el grupo de $\mathrm{MAH}$ - la edad fue significativamente mayor al comparar con el grupo $\mathrm{MAH}+(\mathrm{p}<0,001)$. En cuanto al estado nutricional, 31\%, $43 \%$ y $26 \%$ presentaron normopeso, sobrepeso y obesidad, respectivamente. No se encontraron diferencias significativas en los parámetros antropométricos entre los grupos.

Las mujeres del grupo $\mathrm{MAH}+$ presentaron valores significativamente más elevados de FeS $(p<0,01)$ y ST $(p<0,05)$, y Zpp significativamente más bajos $(p<0,05)$ al comparar con el grupo MAH-. No se encontraron diferencias en el VCM, $\mathrm{Hb}$, TIBC, rTf y FS entre los grupos (tabla 2).

Del total de mujeres evaluadas $(n=81), 3,7 \%(n=3)$ presentó anemia, 28\% $(n=23)$ presentó DFSA y $26 \%(n=21)$ DDF. No se encontraron diferencias significativas en la prevalencia de ADF, DFSA y DDF al comparar los grupos $M A H-$ y $M A H+$ (figura 1). 
Los indicadores del estado nutricional de Cu y $\mathrm{Zn}$ se muestran en la tabla 3. La concentración de CuS, Cp masa y actividad fueron significativamente mayores en el grupo $\mathrm{MAH}+$ $(p<0,001)$ al comparar con el grupo MAH-. No se observaron diferencias en la actividad específica de Cp y ZnS al comparar entre grupos.

En el grupo $M A H-, 3 \%(n=1)$ presentó deficiencia de $\mathrm{Cu}$ y $5 \%(\mathrm{n}=2)$ presentó deficiencia de $\mathrm{Zn}$, mientras que ninguna participante del grupo $\mathrm{MAH}+$ tuvo deficiencia de estos minerales.

En cuanto a la ingesta dietética, el promedio general de ingesta de macronutrientes fue de $2422 \mathrm{kcal}, 100 \mathrm{~g}, 45 \mathrm{~g}$ y 402 g de energía, proteínas, lípidos e hidratos de carbono, respectivamente. A su vez el promedio general de ingesta de micronutrientes fue de 18 mg, 1,9 mg y 11,6 mg de Fe, Cu y Zn, respectivamente. En la tabla 4 se observa la ingesta de macro y micro nutrientes por grupo, no se observaron diferencias en la ingesta de micro y macronutrientes al comparar entre los grupos $\mathrm{MAH}+$ y $\mathrm{MAH}-$.

En el grupo $\mathrm{MAH}+$ y $\mathrm{MAH}-, 3 \%(n=1)$ de cada grupo, presentó ingesta deficiente de $\mathrm{Fe} ; 3 \%(n=1)$ mostró ingesta inadecuada de $\mathrm{Cu}$ en el grupo $\mathrm{MAH}$-, ninguna participante tuvo deficiencia de éste en el grupo $M A H+; 8 \%(n=3)$ presentó ingesta inadecuada de $\mathrm{Zn}$ en el grupo $\mathrm{MAH}-$ y $3 \%(n=1)$ en el grupo $\mathrm{MAH}+$.

\section{DISCUSIÓN}

Los valores de FeS y ST fueron mayores en el grupo MAH+ y significativamente más bajos de Zpp al comparar con el grupo MAH-. El CuS, Cp masa y actividad fueron significativamente mayores en el grupo $\mathrm{MAH}+$.

El MAH puede afectar la concentración de algunos biomarcadores de $\mathrm{Fe}$, Cu y $\mathrm{Zn}(8,9,13)$. Se ha descrito que las mujeres que emplean éste método muestran una disminución de hasta $50 \%$ de las pérdidas de sangre en la menstruación (5), siendo dichas pérdidas el factor más importante que afecta el estado nutricional de Fe y la variación principal del requerimiento nutricional de éste mineral en las mujeres en edad reproductiva $(5,6)$. Nuestros resultados indican que los valores de FeS y ST fueron significativamente mayores en el grupo $\mathrm{MAH}+$ y Zpp fue significativamente menor al comparar con el grupo MAH-. Resultados similares fueron reportados por Frassinelli-Gunderson y cols. (8), quienes mostraron que las mujeres que empleaban MAH presentaban una concentración mayor de FeS y ST, resultados que a su vez, fueron consistentes con otros estudios $(27,28)$. Por otra parte, Larsson y cols. (29)

\section{TABLA 1}

Características generales de mujeres chilenas que emplean o no método anticonceptivo hormonal.

\begin{tabular}{lccc}
\hline & $\begin{array}{c}M A H- \\
(n=40)\end{array}$ & $\begin{array}{c}M A H+ \\
(n=32)\end{array}$ & $P^{*}$ \\
Edad (años) & $36,8 \pm 7,9$ & $29,0 \pm 8,1$ & 0,00 \\
Peso (kg) & $68,6 \pm 10,2$ & $65,2 \pm 8,7$ & 0,14 \\
Talla (m) & $1,56 \pm 0,06$ & $1,57 \pm 0,05$ & 0,40 \\
IMC $\left(\mathrm{kg} / \mathrm{m}^{2}\right)$ & $27,9 \pm 3,9$ & $26,3 \pm 3,8$ & 0,08 \\
\hline $\begin{array}{l}\text { Media } \pm \text { DE } \\
{ }^{*} \text { T - Student de dos colas no pareado }\end{array}$ & & \\
\hline
\end{tabular}

\section{TABLA 2}

Estado nutricional de hierro en mujeres chilenas que emplean o no método anticonceptivo hormonal.

\begin{tabular}{|c|c|c|c|}
\hline & $\begin{array}{l}\text { MAH- } \\
(n=40)\end{array}$ & $\begin{array}{l}\text { MAH+ } \\
(n=32)\end{array}$ & $P^{*}$ \\
\hline $\operatorname{VCM}(f L)^{a}$ & $82,7(75,7-90,5)$ & $84,3(80,7-88,1)$ & 0,13 \\
\hline $\mathrm{Hb}(\mathrm{g} / \mathrm{dL})^{\mathrm{a}}$ & $15,0(13,3-16,8)$ & $15,2(14,2-16,2)$ & 0,26 \\
\hline Zpp $(\mu g / d L G R)^{a}$ & $80,9(57,5-114,0)$ & $72,0(56,1-92,3)$ & 0,04 \\
\hline $\mathrm{FeS}(\mu \mathrm{g} / \mathrm{dL})^{\mathrm{b}}$ & $65,8 \pm 27,1$ & $81,2 \pm 29,0$ & 0,01 \\
\hline $\operatorname{TIBC}(\mu \mathrm{g} / \mathrm{dL})^{\mathrm{a}}$ & $334,2(292,1-382,3)$ & $349,9(292,2-418,9)$ & 0,08 \\
\hline $\mathrm{ST}(\%)^{\mathrm{b}}$ & $20,2 \pm 8,7$ & $23,9 \pm 9,9$ & 0,05 \\
\hline $\mathrm{rTf}(\mathrm{mg} / \mathrm{L})^{\mathrm{a}}$ & $6,7(4,2-10,7)$ & $6,5(4,5-9,4)$ & 0,66 \\
\hline $\mathrm{FS}(\mu \mathrm{g} / \mathrm{L})^{\mathrm{a}}$ & $21,7(10,2-46,3)$ & $22,5(9,8-51,2)$ & 0,42 \\
\hline
\end{tabular}


observaron que después de un periodo de seis meses de uso de MAH se redujo la pérdida menstrual de sangre, sin embargo, no observaron un cambio significativo en la concentración de FS al comparar con los valores observados antes de comenzar a tomar $\mathrm{MAH}$. Concordante con nuestros resultados, dichas investigaciones no han comunicado una mejora en la concentración de $\mathrm{Hb}$, Hto y FS, ni en otros biomarcadores del estatus de Fe en mujeres que usan MAH.

Las diferencias observadas en el FeS, ST y Zpp entre los grupos pueden atribuirse al efecto de la administración de estrógenos sobre el incremento en la síntesis de transferrina y/o sobre la disminución de la pérdida de sangre en la menstruación (29). Por otra parte, la ingesta dietética no explica estos resultados ya que el consumo de Fe fue adecuado para

\section{FIGURA 1}

Prevalencia de deficiencia de Fe según el método anticonceptivo.

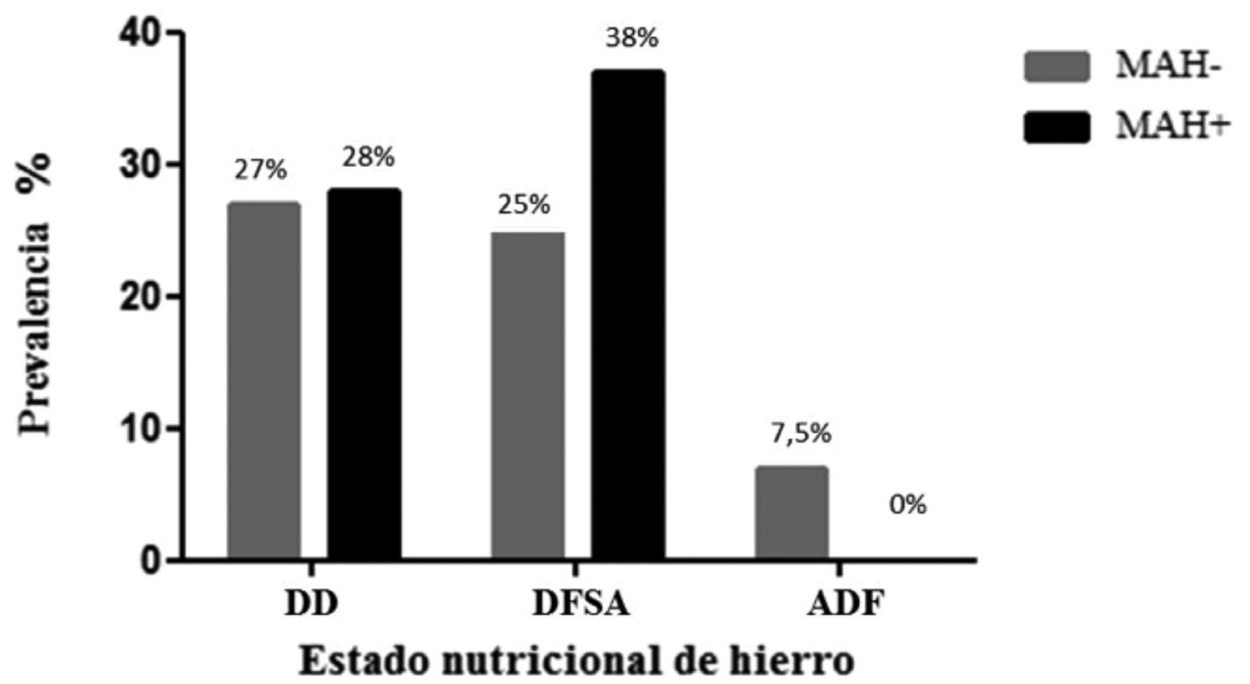

DD: depósitos depletados. DFSA: Deficiencia de Fe sin anemia. ADF: Anemia por deficiencia de Fe.

TABLA 3

Estado nutricional de cobre y zinc en mujeres chilenas que emplean o no método anticonceptivo hormonal.

\begin{tabular}{|c|c|c|c|}
\hline & $\begin{array}{l}\text { MAH- } \\
(n=40)\end{array}$ & $\begin{array}{l}\text { MAH+ } \\
(n=32)\end{array}$ & $P^{*}$ \\
\hline CuS & 112,9 & 152,6 & 0,00 \\
\hline$(\mu \mathrm{g} / \mathrm{dL})^{\mathrm{a}}$ & $(91,9-138,5)$ & $(109,3-213,2)$ & \\
\hline \multirow[t]{2}{*}{ Cp masa $(\mathrm{mg} / \mathrm{dL})^{\mathrm{a}}$} & 47,9 & 58,3 & 0,00 \\
\hline & $(41,1-56,1)$ & $(44,1-77,1)$ & \\
\hline \multirow[t]{2}{*}{$\mathrm{Cp}$ actividad $(\mathrm{mg} / \mathrm{dL})^{\mathrm{a}}$} & 18,4 & 23,0 & 0,00 \\
\hline & $(14,2-23,8)$ & $(17,3-30,5)$ & \\
\hline Actividad específica de $C p^{b}$ & $0,39 \pm 0,1$ & $0,4 \pm 0,1$ & 0,32 \\
\hline \multirow[t]{2}{*}{$\mathrm{ZnS}(\mu \mathrm{g} / \mathrm{dL})^{\mathrm{a}}$} & 82,9 & 84,5 & 0,50 \\
\hline & $(72,7-94,4)$ & $(66,2-108,0)$ & \\
\hline $\begin{array}{l}\text { Promedio Geométrico y rango de } \pm 1 \mathrm{DE} \\
\text { Media } \pm \mathrm{DE} \\
\mathrm{T}-\text { Student de una cola no pareado. }\end{array}$ & & & \\
\hline
\end{tabular}


ambos grupos y no se observaron diferencias significativas en la ingesta de éste mineral. A su vez, estudios de absorción con isótopos estables no han mostrado que exista diferencia en la absorción intestinal de Fe, Cu y Zn según el MA (30).

La prevalencia de anemia observada en el total de la muestra de este estudio correspondió a 3,7\% en comparación con lo reportado en la Encuesta Nacional 2003 que fue de $5,1 \%$ (3), lo que puede sugerir que la anemia ha dejado de ser un problema de salud en Chile según la clasificación de la Organización Mundial de la Salud (20). Sin embargo, la prevalencia de DFSA y DDF continúa siendo elevada según lo indicado en esta investigación, para lo cual se requieren nuevas estrategias enfocadas a la disminución de la prevalencia de deficiencia de Fe en etapas previas a la anemia.

Al comparar la prevalencia de DDF, DFSA y ADF entre los grupos $\mathrm{MAH}+$ y $\mathrm{MAH}-$, no se encontró una diferencia significativa, a pesar que existieron variaciones en algunos biomarcadores. Estos resultados pueden deberse a que en mujeres con una buena ingesta de hierro, la reducción de hasta $50 \%$ de la pérdida de sangre en la menstruación por el uso anticonceptivos hormonales (31), no sería suficiente para establecer diferencias en el estatus de este mineral. Un estudio realizado en 2377 mujeres en base a datos del Estudio Nacional de Salud y Nutrición (NAHNES) de 1988 - 1994, mostró que en mujeres blancas no hispanas la prevalencia de ADF no se asociaba con el tipo de anticonceptivo a diferencia de lo reportado en mujeres mexicanas donde el tipo de anticonceptivo se asoció a la prevalencia de anemia (32). Es posible que en países donde la deficiencia de micronutrientes es baja, el uso de $\mathrm{MAH}$ no tenga una implicancia biológicamente importante en la mejora de los depósitos de $\mathrm{Fe}$, ni en la prevalencia de anemia como hace varias décadas mostraba literatura $(7,31)$. Estudios en los que se observaba que la concentración de $\mathrm{Hb}$ era mayor en las mujeres que utilizaban $\mathrm{MAH}$, son de la década de los 60, a partir de esa época se han implementado cambios enfocados a mejorar la nutrición de Fe de la población en general. A partir de 1967 se implementó en Chile la ley de fortificación de harina de trigo con Fe $(30 \mathrm{mg} / \mathrm{kg}$ ) y a su vez con el paso del tiempo la oferta de alimentos fortificados se ha incrementado,33 por lo que es probable que la mejora en el estado nutricional de Fe es mujeres chilenas en edad reproductiva no se debiera solo al tipo de anticonceptivo empleado.
Por otra parte, en cuanto al estatus de Cu, la concentración de CuS, Cp masa y actividad fueron significativamente mayores en el grupo con $\mathrm{MAH}+$, lo cual es consistente con otros estudios $(9,10,11)$, y puede atribuirse al efecto de los estrógenos sobre la síntesis hepática de Cp masa, así mismo, incrementa la concentración de CuS dado que existe una alta correlación entre ambos biomarcadores (34). No existe suficiente certeza sobre la implicancia biológica de la elevación de las concentraciones de $\mathrm{Cu}$, sin embargo se ha (35). La actividad específica de Cp mostró ser un buen indicador del estado nutricional de Cu debido a que no se vio afectada por terapia hormonal (11). Esto sugiere que el empleo de $M A H$, altera las concentraciones de biomarcadores de $\mathrm{Cu}$, pero no el estado nutricional del mismo.

Respecto a la nutrición del Zn, algunos estudios de la década de los 80 indicaban que la administración de $M A H$ podía alterar el ZnS por medio de una disminución de albúmina (14), proteína que transporta $75 \%$ del ZnS. A su vez mostraban que el ZnS disminuía por el empleo de $M A H$, sin embargo, la concentración de $\mathrm{Zn}$ en eritrocito incrementaba (36). En dichos estudios la ingesta dietética de Zn no se evaluó. Una investigación más reciente realizada en 330 mujeres de Nueva Zelanda (39\% empleaba MAH), indicó que en este grupo la concentración de ZnS era significativamente menor (13), pero al mismo tiempo mostraron que la ingesta de $\mathrm{Zn}$, que cumplía con los criterios de adecuación, había disminuido y la prevalencia de deficiencia había incrementado al comparar con años anteriores. Contrario a esto, en nuestro estudio no existieron diferencias en la concentración de ZnS al comparar entre los grupos $\mathrm{MAH}$ - y $\mathrm{MAH}+$, y el consumo dietético de Zn fue similar en estos grupos y a su vez adecuado. Similares resultados fueron mostrados por Hinks y cols. (10) quienes reportaron que el empleo de $\mathrm{MAH}$ no afectaba $\mathrm{ZnS}$.

La Encuesta Nacional de Salud del año 2009 - 2010 (37), indicó que en mujeres de 25 a 44 años, 35,6\% y 28,3\% presentaron sobrepeso y obesidad, respectivamente, cifras similares a las que se observaron en este estudio, en el que $43 \%$ de las mujeres presentó sobrepeso y $26 \%$ obesidad. Esto indica lo alarmante de este problema de salud.

Es importante recalcar, que los cambios observados en los indicadores del estatus de $\mathrm{Fe}, \mathrm{Zn}$ y $\mathrm{Cu}$ asociados al empleo de MAH no modificaron la prevalencia de deficiencia de estos

\section{TABLA 4}

Ingesta dietética de macro y micronutrientes en mujeres chilenas que emplean o no método anticonceptivo hormonal.

\begin{tabular}{|c|c|c|c|}
\hline & $\begin{array}{l}\text { MAH- } \\
(n=40)\end{array}$ & $\begin{array}{l}\text { MAH+ } \\
(n=32)\end{array}$ & $P^{*}$ \\
\hline Energía (Kcal) & $2375 \pm 686,4$ & $2482 \pm 804$ & 0,54 \\
\hline Proteínas (g) & $97,9 \pm 28,4$ & $102,7 \pm 38,9$ & 0,54 \\
\hline Lípidos (g) & $42,1 \pm 21,5$ & $49,4 \pm 18,5$ & 0,13 \\
\hline $\mathrm{HC}(\mathrm{g})$ & $402,2 \pm 115,2$ & $404 \pm 143,1$ & 0,95 \\
\hline Hierro (mg) & $17,8 \pm 5,6$ & $18,3 \pm 7,7$ & 0,7 \\
\hline Zinc (mg) & $11,1 \pm 3,4$ & $12,3 \pm 5,1$ & 0,27 \\
\hline Cobre (mg) & $1,7 \pm 0,5$ & $2,0 \pm 1,1$ & 0,08 \\
\hline
\end{tabular}


minerales, posiblemente debido a que en mujeres chilenas la prevalencia de deficiencia de estos micronutrientes es baja. Sería importante estudiar lo que ocurre en una población en la que la carencia de estos minerales sea un problema relevante de salud.

\section{CONCLUSIÓN}

Las mujeres que empleaban un método anticonceptivo de tipo hormonal mostraron valores más elevados de hierro sérico, saturación de transferrina, cobre sérico, ceruloplasmina masa y actividad y menor concentración de zinc, protoporfirina al comparar con aquellas que no usaban. Sin embargo, esto no parece tener un efecto en la disminución de la prevalencia de la deficiencia de Fe en sus diferentes etapas en mujeres chilenas, posiblemente en países donde la prevalencia de micronutrientes sea mayor; el uso de método anticonceptivo de tipo hormonal pueda tener un efecto significativo.

\section{RESUMEN}

Las mujeres en edad reproductiva conforman un grupo vulnerable de presentar deficiencia de micronutrientes. Se ha relacionado el método anticonceptivo (MA) con el estatus de hierro (Fe), y al MA hormonal (MAH) con la alteración de biomarcadores de cobre (Cu) y zinc ( $\mathrm{Zn}$ ). El objetivo del presente estudio fue evaluar el efecto del MA sobre los biomarcadores del estatus de $\mathrm{Fe}$, Cu y Zn y la prevalencia de deficiencia de éstos, en 81 mujeres chilenas en edad reproductiva. Se evaluó el volumen corpuscular medio (VCM), hemoglobina ( $\mathrm{Hb})$, Zinc protoporfirina (Zpp), Fe sérico (FeS), saturación de tranferrina (ST), capacidad total de unión de Fe (TIBC), receptor de transferrina ( $r T f)$, ferritina sérica (FS), cobre sérico (CuS), ceruloplasmina (Cp) masa, actividad y actividad específica y zinc sérico (ZnS). El 40\% utilizó MAH y 49\% no utilizaba $\mathrm{MAH}$. Las mujeres que utilizaban $\mathrm{MAH}$ presentaron valores más altos de FeS, ST, CuS, Cp masa y actividad $(p<0,05)$ y menor Zpp $(p<0,05)$ que aquellas que no utilizaban $M A H$. No se encontraron diferencias en el VCM, Hb, TIBC, rTf, FS, ZnS, y $\mathrm{Cp}$ actividad específica entre ambos grupos. Se concluye que el uso de MAH no afectó el estatus de $\mathrm{Fe}, \mathrm{Cu}$ y $\mathrm{Zn}$ en la población estudiada.

Palabras clave: Hierro, cobre, zinc, método anticonceptivo, mujeres en edad reproductiva.

Agradecimientos: Este estudio fue financiado por el Fondo Nacional del Desarrollo Científico y Tecnológico (FONDECYT \# 1133075).

\section{BIBLIOGRAFÍA}

1. Olivares M, Castillo Durán C, Uauy R. Cobre y cinc. En: Gil A, editor. Tratado de Nutrición. 2a Edición. Editorial Médica Panamericana, Madrid, 2010, Tomo I, Capítulo 28, p. 687-704.

2. Olivares M, Arredondo M, Pizarro F. Hierro. Gil A, editor. Tratado de Nutrición 2a Edición. Editorial Médica Panamericana, Madrid, 2010, Tomo I, Capítulo 27, p. 668-86.

3. MINSAL. Encuesta Nacional de Salud 2003. Accesada online el 18 de febrero 2014 en http://epi.minsal.cl/epi/ html/invest/ens/informefinalens.pdf.

4. Cediel G, Olivares M, Araya M, Letelier A, López de Romaña $D$, Pizarro $F$. Efecto de la inflamación subclínica sobre el estado nutricional de hierro, cobre y zinc en adultos. Rev Chil Nutr. 2009; 36: 8 - 14.

5. Hallberg L, Rossander - Hulten L- Iron requirements in menstruating women. Am J Clin Nutr. 1991; 54: 1047- 58.
6. Harvey L, Armah C, Dainty J, Foxall R, Lewis D, Langford $N$, Fairweather - Trait S. Impact of menstrual blood loss and diet on iron deficiency among women in the UK. Brit J Nutr. 2005; 94: 557 - 64.

7. Zadeh J, Karabus C, Fielding J. Haemoglobin concentration and other values in women using an intrauterine device or taking corticosteroid contraceptive pills. Brit Med J. 1967; 4: $708-11$.

8. Frassinelli-Gunderson E, Margen S, Brow J. Iron stores in users of oral contraceptive agents. Am J Clin Nutr. 1985; 41: $703-12$.

9. Carruthers M, Hobbs $C$, Warren R. Raised serum copper and ceruloplasmin levels in subjects taking oral contraceptives J Clin Pathol. 1966; 19: 498 - 500.

10. Hinks L, Clayton B, Lloyd R. Zinc and copper concentrations in leucocytes and erythrocytes in healthy adults. J Clin Pathol.1983; 36: 1016 - 21.

11. Olivares M, Méndez MA, Astudillo PA, Pizarro F. Present situation of biomarkers for copper status. Am J Clin Nutr. 2008; 88: 859S - 62S

12. Milne D, Johnson P. Assessment of Copper Status: Effect of age and gender on reference ranges in healthy adults. Clin Chem. 1993; 39: $883-7$.

13. Gibson R, Heath A, Limbaga M, Prosser N, Skeaff $M$. Are changes in food consumption patterns associated with lower biochemical zinc status among women from Dunedin, New Zealand? Brit J Nutr. 2001; 86: 71 - 80.

14. King J. Do women using oral contraceptive agents require extra zinc?. J Nutr. 1987; 117: 217 - 9.

15. Ministerio de Salud. Segunda Encuesta de calidad de vida y salud Chile 2006. Accesada online el 2 de abril 2014 en http://epi.minsal.cl/wp-content/uploads/2012/07/Informe-Final-Encuesta-de-Calidad-de-Vida-y-Salud-2006. pdf.

16. Norton K, Whittingham, Carter Linsay, Kerr Deborah, Gore C, Marfell-Jones M. Técnicas de medición en antropometría. In: Norton $K$, Olds $T$ editors. Antropometrica (Edición en español: Mazza J) Argentina: Biosystem Servicio Educativo Press 2000: 23 - 60.

17. Fischer DS, Price DC. A simple serum iron method using the new sensitive chromogen tripyridil-s-triazine. Clin Chem. 1964; 10: 21 - 31

18. International Anemia Consultative Group (INACG). Measurements of iron status. Washington: Nutr Found. 1985: 35-54.

19. Ravin A. An improved colorimetric enzymatic assay for ceruloplasmin. J Lab Clin Med. 1961; 58: 161 - 168.

20. Report of a joint WHO/UNICEF/UNU expert consultation. Iron deficiency anaemia: assessment, prevention, and control: a guide for programme managers. Geneva, Switzerland: WHO, 2001.

21. Center for Disease Control and Prevention. Recommendations to prevent and control iron deficiency in the United States. MMWR. 1998; 47:1-29.

22. Skikne $B$, Flowers $C$, Cook J. Serum transferrin receptor: a quantitative measure of tissue iron deficiency. Blood 1990; 75: $1870-76$.

23. Elin RJ. Reference intervals and laboratory values. In: Cecil Textbook of Medicine. Goldman L, Ansiello DA, editors. Philadelphia, PA: WB Saunders; 2004: 2496 - 05.

24. Schmidt-Hebbel H, Pennacchiotti I, Masson L, Mella MT. Tabla de composición química de los alimentos chilenos. Santiago, Chile: Facultad de Ciencias Químicas y Farmacéuticas, Departamento de Ciencia de los Alimentos y 
Tecnología Química, Universidad de Chile, 1992.

25. Olivares M, Pizarro F, De Pablo S, Araya M, Uauy R. Iron, zinc and copper: contents in common Chilean foods and daily intakes in Santiago City, Chile. Nutrition 2004; 20: $205-12$.

26. United States Department of Agriculture. USDA national nutrient database for standard reference, release 24; 2012. Accesada online el 25 Enero 2014 en http://www.ars.usda. gov/Services/docs. htm?docid $=22808$

27. Burton J, Manc B. Effect of oral contraceptive on haemoglobin, packed cell volumen, seum - iron, and total iron - binding capacity in healthy women. Lancet 1967; 289: $978-80$

28. Margen S, King J. Effect of oral contraceptive agents on the metabolism of some trace minerals. Am J Clin Nutr. 1975; 28 : $392-402$.

29. Larsson G, Milsom L, Lindstedt $G$, Rybo G. The influence of a low-dose combined oral contraceptive on menstrual blood loss and iron status. Contraception 1992; 46: 327 - 34.

30. King J, Raynols W, Margen S. Absorption of stable isotopes of iron, copper, and zinc during oral contraceptive use. Am J Clin Nutr. 1978; 31: 1198 - 03.

31. Hallberg L, Bengtsson C, Garby L, Lennartsson J, Rossander
L, Tibblin E. An analysis of factors leading to a reduction in iron deficiency in Swedish women. Bull WHO 1979; 57: $947-54$.

32. Frith-Terhune A, Cogswell M, Kettel L, Will J, Ramakrishnan $U$. Iron deficiency anemia: higher prevalence in Mexican American than in non-Hispanic white females in the third National Health and Nutrition Examination Survey, 1988-1994. Am J Clin Nutr. 2000; 72: 963 - 8.

33. Olivares $M$, Pizarro F, Hertrampf E, Walter T, Arredondo $M$, Letelier A. Fortificación de alimentos con hierro en Chile. Rev Chil Nutr. 2000; 27: 340 - 4.

34. Arredondo M, González M, Olivares M, Pizarro F, Araya M. Ceruloplasmin, an indicator of copper status. Biol Trace Elem Res. 2008; 123:261-9.

35. Linder MC, Hazegh-Azam M. Copper biochemestry and molecular biology. Am J Clin Nutr. 1996; 63: 297S-811S.

36. Prasad A, Oberleas D, Lei K, Moghissi K, Stryker J. Effect of oral contraceptive agents on nutrients: I Minerals. Am J Clin Nutr. 1975; 28: $377-84$.

37. MINSAL. Encuesta Nacional de Salud 2009 - 2010. Accesada online el 17 de junio 2014 en http://epi.minsal.cl/ wp-content/uploads/2012/07/Informe-ENS-2009-2010.CAP-5_FINALv1julioccepi.pdf 